\title{
Magnetic field variations associated with SES
}

The instrumentation used for investigating their detectability

\author{
By Panayiotis Varotsos, ${ }^{\dagger \dagger}$ Nikos SARLIS, and Efthimios Skordas
}

Solid Earth Physics Institute, University of Athens, Panepistimiopolis, Zografos 157 84, Athens, Greece

(Communicated by Seiya UyEDA, M. J. A., June 12, 2001)

\begin{abstract}
Small amplitude Seismic Electric Signals (SES), are not accompanied by readily detectable variations of the horizontal components of the magnetic field. This does not hold for strong SES activities, i.e., before earthquakes with $\mathrm{M} \sim 6.5$ at epicentral distances of $\sim 100$ [km]. The instrumentation used to obtain such conclusions is described. Attention is focused on a careful calibration of the coil magnetometers that were in operation at Ioannina station when the strong SES activities associated with the 6.6 earthquake at Grevena-Kozani were recorded. These magnetometers act as $\mathrm{dB} / \mathrm{dt}$ detectors for periods longer than around half a second; furthermore their output is "neutralized" after 200 [ms] from the "arrival" of a fast step magnetic variation. In other words, a signal recorded by these magnetometers should correspond to a magnetic variation that has "arrived" at the sensor less than 200 [ms] before the recording. This property is critical for the study of the time difference between the variations in the electric and the magnetic fields. The variations in these fields "arrive" simultaneously (within experimental accuracy) from the usual nearby artificial sources, while for magnetotellurics the magnetic recordings "precede" those of the electric field. Both behaviors differ from that observed in the case of strong SES activities, a matter described in the accompanying paper. ${ }^{1)}$
\end{abstract}

Key words: Magnetic measurements; electrical measurements; SES.

Introduction. It has long been reported (see ref. 2) and references therein) that small amplitude SES, e.g., those corresponding to magnitude $\mathrm{M} \sim 5.5$ earthquakes (EQ) at epicentral distances ( $r$ ) of the order of $100[\mathrm{~km}]$, are not accompanied by easily observable variations of the horizontal components of the magnetic field. On the other hand, theoretical calculations ${ }^{3)-6 \text { ) }}$ indicate that strong SES activities, e.g., those related with EQ of magnitude $\mathrm{M} \sim 6.5 \mathrm{EQ}$ at $\mathrm{r} \sim 100[\mathrm{~km}]$, should be accompanied by detectable magnetic field variations, e.g., $\mathrm{B} \sim 10^{-1}[\mathrm{nT}]$. This was actually observed at Ioannina (IOA) for the $\mathrm{M} \sim 6.6 \mathrm{EQ}$ that occurred at Grevena-Kozani on May 13, 1995. ${ }^{7,8)}$

The present paper first deals with the instrumentation used at IOA, because it is essential to understand which physical quantity (e.g., $B$ or $\mathrm{dB} / \mathrm{dt}$ ) is measured in each frequency range. In the second part, we give examples of recordings of nearby artificial noises and magnetotelluric (MT) variations. The magnetic field

\footnotetext{
†) Correspondence to: P. Varotsos.
}

variations that accompanied the strong SES activities preceding the 6.6 EQ at Grevena-Kozani are described in the accompanying paper. ${ }^{1)}$

Calibration of the magnetometers used. Permanent recording of the magnetic field variations at IOA at the time of the Grevena-Kozani EQ was carried out by a three component DANSK coil magnetometer (DMM). Furthermore, the mobile MT-1 sounding system of Electro-Magnetic Instruments (EMI) has been in use since 1996, either for temporary MT measurements at several sites in Epirus area or, occasionally, for several months' continuous operation at IOA. Besides a laboratory calibration, ${ }^{9)}$ an in situ (i.e., at IOA) calibration was performed by comparing the DMM recordings with those of the EMI-magnetometers and relying on the accurate laboratory calibration of the latter by the manufacturer. ${ }^{10)}$ A sandwich technique was employed in which each DMM sensor was located parallel to and between two parallel EMI coil sensors (EMM). Such a deployment assures that when we have the same recordings in the two EMM, the DMM should lie in the 

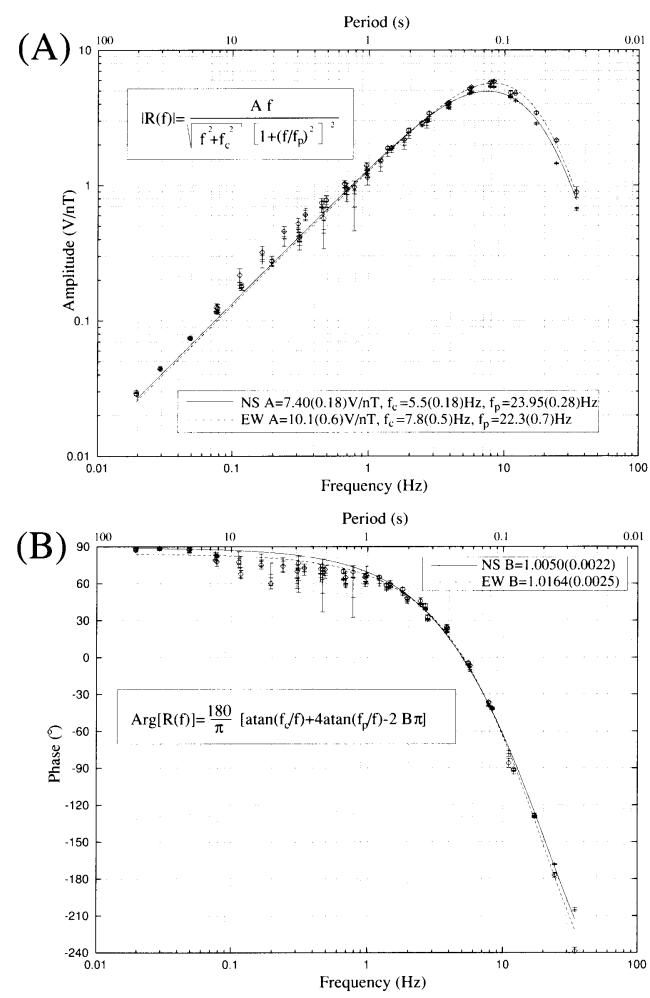

Fig. 1. The experimental results for the frequency response, amplitude (A) and phase (B), of the two horizontal DMM along with the fitting curve for $R(f)$.

same magnetic field (cf. the distance between DMM and EMM was only around $7[\mathrm{~m}]$ ). The output of the three coil sensors (2 EMM and 1 DMM) was the input to the MT-1 data acquisition system, ${ }^{10)}$ which allowed us to record magnetic variations for a time period corresponding to a data collection of approximately 5 Mbytes.

The data were analyzed using the Mtr95 computer program of EMI, which has been designed for the interpretation of MT-1 data. Only those data blocks with a coherency of $\geq 0.99$ between the two EMM have been used. The spectrum analysis of the transfer function T(f) between DMM and the two EMM was made as follows: upon assuming for the DMM a fictitious response $\mathrm{R}_{\mathrm{BF} 4-9511}(\mathrm{f})$ (i.e., that of EMI magnetic sensor BF49511), we then obtain the data shown in Fig. 1 by multiplying the fictitious response by the transfer function obtained experimentally, $R_{D M M}(f)=T(f) R_{B F 4-9511}(f)$. Since several measurements have been carried out for each frequency, the results shown in Fig. 1 display the mean value along with the standard error of the mean.

Next, we fitted both the field DMM data and the lab-
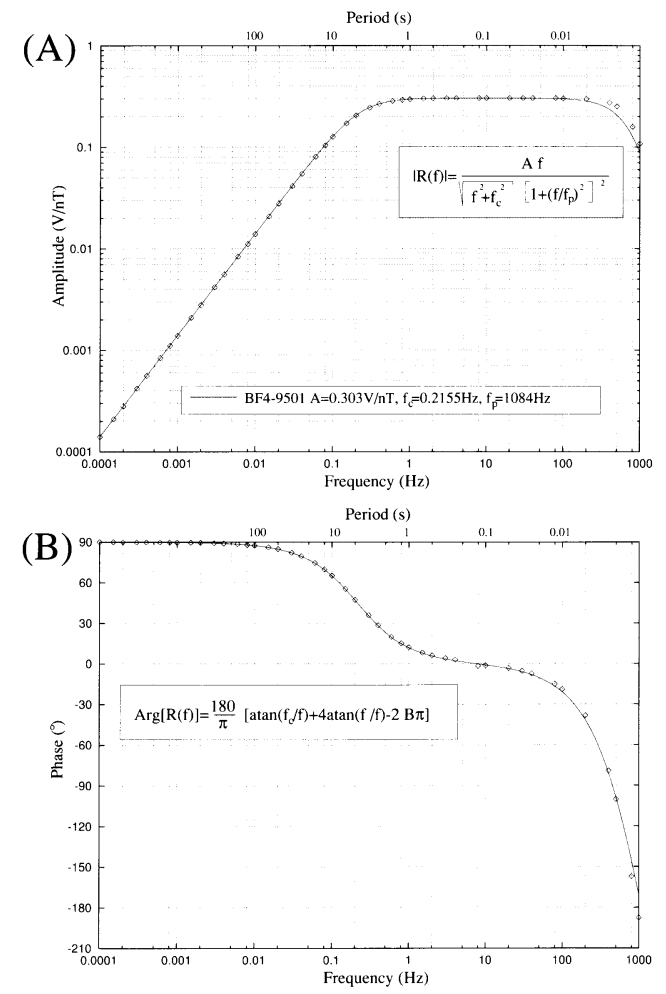

Fig. 2. The laboratory calibration by EMI for the frequency response, amplitude (A) and phase (B), of the EMM (BF4-9501 sensor) along with the fitting curve for $R(f)$.

oratory EMM data (the latter provided by EMI ${ }^{10)}$ ), using a general analytic expression for the frequency response R(f) (see Figs. 1 and 2, respectively)

$$
\mathrm{R}(\mathbf{f}): \mathrm{V}(\mathbf{f})=\mathrm{R}(\mathbf{f}) \mathrm{B}(\mathbf{f}),
$$

where $V(f)$ is the voltage response of the magnetometer and $B(f)$ the magnetic field at a frequency $f$. Assuming that R(f) is the standard coil response Af/ $\left(\mathrm{f}-\mathrm{jf} \mathrm{f}_{\mathrm{c}}\right)$ multiplied by the response $1 /\left(\mathrm{f} / \mathrm{f}_{\mathrm{p}}-\mathrm{j}\right)^{4}$ of a fourth order low pass filter, we have:

$$
R(f)=\frac{A f}{\left.\left(f-j f_{c}\right)\left(f / f_{p}\right)-j\right)^{4}} .
$$

The symbol A stands for an amplitude in $\mathrm{V} / \mathrm{nT}, \mathrm{j}$ the imaginary unit, $\mathrm{f}_{\mathrm{c}}$ a frequency related to the decay time constant $\tau_{\mathrm{d}}\left(\equiv 1 /\left(2 \pi \mathrm{f}_{\mathrm{c}}\right)\right)$ of the coil, and $\mathrm{f}_{\mathrm{p}}$ the cut-off frequency of a simple fourth order low-pass filter of DMM. The latter frequency is related to the rise time constant $\tau_{\mathrm{r}}$ of the magnetometer through $\tau_{\mathrm{r}} \equiv 1 /\left(2 \pi \mathrm{f}_{\mathrm{p}}\right)$. The expression of eq. [2] was applied to both amplitude and 
Table I. The values of the fitting parameters for R(f) along with the decay and the rise time constants $\tau_{\mathrm{d}}, \tau_{\mathrm{r}}$ for the two types of coil magnetometers

\begin{tabular}{lccccccc}
\hline Type of magnetometer & \multicolumn{2}{c}{$\mathrm{A}(\mathrm{V} / \mathrm{nT})$} & \multicolumn{2}{c}{$\mathrm{f}_{\mathrm{c}}(\mathrm{Hz})$} & $\mathrm{f}_{\mathrm{p}}(\mathrm{Hz})$ & $\tau_{\mathrm{d}}(\mathrm{ms})$ & \multicolumn{1}{c}{$\tau_{\mathrm{r}}(\mathrm{ms})$} \\
\hline DANSK(DMM) NS & $7.40 \quad \pm 0.18$ & $5.50 \quad \pm 0.18$ & $23.95 \pm 0.28$ & $28.9 \pm 0.9$ & 6.65 & \pm 0.08 \\
DANSK(DMM) EW & 10.1 & \pm 0.6 & $7.8 \quad \pm 0.5$ & $22.3 \pm 0.7$ & $20.4 \pm 1.3$ & 7.14 & \pm 0.22 \\
EMI(EMM) & $0.3030 \pm 0.0001$ & $0.2155 \pm 0.0001$ & $1084.0 \pm 0.5$ & $738.5 \pm 0.3$ & $0.14682 \pm 0.00014$ \\
\hline
\end{tabular}

phase, and a non-linear least squares fitting was performed using the constant chi-square $(p=95 \%)$ boundaries for the determination of the errors in the fitting parameters. ${ }^{11)}$ The corresponding values are shown on Table I, for both coil magnetometers, i.e., DMM and EMM.

We can evaluate the expressions for the impulse response function $\mathrm{I}(\mathrm{t})$,

$$
\begin{aligned}
& I(t)=\int_{-\infty}^{\infty} R(f) \exp (2 \pi f t j) d f: \\
& V(t)=\int_{-\infty}^{\infty} I(\xi) B(t-\xi) d \xi
\end{aligned}
$$

the Heaviside (or unit step) response function $\mathrm{H}(\mathrm{t})$,

$$
\begin{aligned}
& \mathrm{H}(\mathrm{t})=\int_{-\infty}^{\infty} \frac{\mathrm{R}(\mathrm{f})}{2 \pi \mathrm{fj}} \exp (2 \pi \mathrm{ftj}) \mathrm{df}: \\
& \mathrm{V}(\mathrm{t})=\int_{-\infty}^{\infty} \mathrm{H}(\xi) \frac{\mathrm{dB}}{\mathrm{dt}}(\mathrm{t}-\xi) \mathrm{d} \xi,
\end{aligned}
$$

and the ramp response function $\mathrm{P}(\mathrm{t})$,

$$
\mathrm{P}(\mathrm{t})=\int_{-\infty}^{\mathrm{t}} \mathrm{H}(\xi) \mathrm{d} \xi
$$

which describes the output of the magnetometer in the case of a ramp (linearly increasing with time) magnetic field of the form $B(t)=t \Theta(t)[n T / s]$, where $\Theta(x)$ is the Heaviside unit step function, i.e., $\Theta(x)=1$ if $x>0$, $\Theta(\mathrm{x})=0$ elsewhere. Using eq. [2], we can evaluate eqs. [3], [4] and [5] by contour integration on the complex $\mathrm{f}$ plane. The following expressions are obtained for $t>0$ :

$$
\begin{aligned}
\mathrm{I}(\mathrm{t}) & =\mathrm{A} \frac{\tau_{\mathrm{d}}^{3}}{\left(\tau_{\mathrm{d}}-\tau_{\mathrm{r}}\right)^{4}}\left(\mathrm{e}^{\left.-\mathrm{t} / \tau_{\mathrm{r}}-\mathrm{e}^{-\mathrm{t} / \tau_{\mathrm{d}}}\right)}\right. \\
& +\mathrm{A} \frac{\tau_{\mathrm{d}}^{2}}{\left(\tau_{\mathrm{d}}-\tau_{\mathrm{r}}\right)^{3}} \frac{\mathrm{t}}{\tau_{\mathrm{r}}} \mathrm{e}^{-\mathrm{t} / \tau_{\mathrm{r}}} \\
& +\frac{\mathrm{A}}{2} \frac{\tau_{\mathrm{d}}}{\left(\tau_{\mathrm{d}}-\tau_{\mathrm{r}}\right)^{2}}\left(\frac{\mathrm{t}}{\tau_{\mathrm{r}}}\right)^{2} \mathrm{e}^{-\mathrm{t} / \tau_{\mathrm{r}}} \\
& +\frac{\mathrm{A}}{6} \frac{\tau_{\mathrm{d}}}{\left(\tau_{\mathrm{d}}-\tau_{\mathrm{r}}\right) \tau_{\mathrm{r}}}\left(\frac{\mathrm{t}}{\tau_{\mathrm{r}}}\right)^{3} \mathrm{e}^{-\mathrm{t} / \tau_{\mathrm{r}}}
\end{aligned}
$$

$$
\begin{aligned}
\mathrm{H}(\mathrm{t}) & =\mathrm{A} \frac{\tau_{\mathrm{d}}^{4}}{\left(\tau_{\mathrm{d}}-\tau_{\mathrm{r}}\right)^{4}}\left(\mathrm{e}^{\left.-\mathrm{t} / \tau_{\mathrm{d}}-\mathrm{e}^{-\mathrm{t} / \tau_{\mathrm{r}}}\right)}\right. \\
& -\mathrm{A} \frac{\tau_{\mathrm{d}}^{3}}{\left(\tau_{\mathrm{d}}-\tau_{\mathrm{r}}\right)^{3}} \frac{\mathrm{t}}{\tau_{\mathrm{r}}} \mathrm{e}^{-\mathrm{t} / \tau_{\mathrm{r}}} \\
& -\frac{\mathrm{A}}{2} \frac{\tau_{\mathrm{d}}^{2}}{\left(\tau_{\mathrm{d}}-\tau_{\mathrm{r}}\right)^{2}}\left(\frac{\mathrm{t}}{\tau_{\mathrm{r}}}\right)^{2} \mathrm{e}^{-\mathrm{t} / \tau_{\mathrm{r}}} \\
& -\frac{\mathrm{A}}{6} \frac{\tau_{\mathrm{d}}}{\left(\tau_{\mathrm{d}}-\tau_{\mathrm{r}}\right)}\left(\frac{\mathrm{t}}{\tau_{\mathrm{r}}}\right)^{3} \mathrm{e}^{-\mathrm{t} / \tau_{\mathrm{r}}}
\end{aligned}
$$

and

$$
\begin{aligned}
\mathrm{P}(\mathrm{t}) & =\mathrm{A} \frac{\tau_{\mathrm{d}}^{5}}{\left(\tau_{\mathrm{d}}-\tau_{\mathrm{r}}\right)^{4}}\left(1-\mathrm{e}^{-\mathrm{t} / \tau_{\mathrm{d}}}\right) \\
& -\mathrm{A} \frac{\tau_{\mathrm{d}}^{4} \tau_{\mathrm{r}}}{\left(\tau_{\mathrm{d}}-\tau_{\mathrm{r}}\right)^{4}}\left(1-\mathrm{e}^{\left.-\mathrm{t} / \tau_{\mathrm{r}}\right)}\right. \\
& -\mathrm{A} \frac{\tau_{\mathrm{d}}^{3} \tau_{\mathrm{r}}}{\left(\tau_{\mathrm{d}}-\tau_{\mathrm{r}}\right)^{3}}\left|1-\left(1+\frac{\mathrm{t}}{\tau_{\mathrm{r}}}\right) \mathrm{e}^{-\mathrm{t} / \tau_{\mathrm{r}}}\right| \\
& -\frac{\mathrm{A}}{2} \frac{\tau_{\mathrm{d}}^{2} \tau_{\mathrm{r}}}{\left(\tau_{\mathrm{d}}-\tau_{\mathrm{r}}\right)^{2}}\left\{2-\left|2+2 \frac{\mathrm{t}}{\tau_{\mathrm{r}}}+\left(\frac{\mathrm{t}}{\tau_{\mathrm{r}}}\right)^{2}\right| \mathrm{e}^{-\mathrm{t} / \tau_{\mathrm{r}}}\right\} \\
& -\frac{\mathrm{A}}{6} \frac{\tau_{\mathrm{d}} \tau_{\mathrm{r}}}{\left(\tau_{\mathrm{d}}-\tau_{\mathrm{r}}\right)}\left\{6-\left|6+6 \frac{\mathrm{t}}{\tau_{\mathrm{r}}}+3\left(\frac{\mathrm{t}}{\tau_{\mathrm{r}}}\right)^{2}+\left(\frac{\mathrm{t}}{\tau_{\mathrm{r}}}\right)^{3}\right| \mathrm{e}^{-\mathrm{t} / \tau_{\mathrm{r}}}\right\},
\end{aligned}
$$

whereas $I(t)=H(t)=P(t)=0$ for $t<0$. Fig. 3 depicts the impulse, Heaviside and ramp responses for the two magnetometers, the DMM and the EMM respectively, while Table I shows their corresponding decay and rise time constants $\tau_{\mathrm{d}}, \tau_{\mathrm{r}}$.

On the basis of eqs. [6], [7] and [8] and Fig. 3 (A), (B), (C), the operation of the two coil magnetometers may be interpreted as follows: Firstly, from eq. [6] for the impulse response function $\mathrm{I}(\mathrm{t})$ and after recalling its definition given in eq. [3], we conclude that the output of each magnetometer at a given time $t$ is proportional to the difference of the magnetic field functionals $\Phi_{\tau_{\mathrm{r}}}[\mathrm{B}]$ and $\Phi_{\tau_{d}}[B]$, divided by the time difference $\tau_{d}-\tau_{r}$; these two functionals weight the magnetic field $\mathrm{B}(\mathrm{t})$ with appropriate exponentially decreasing functions of time constants $\tau_{\mathrm{r}}$ and $\tau_{\mathrm{d}}$ respectively. More precisely, we obtain: 

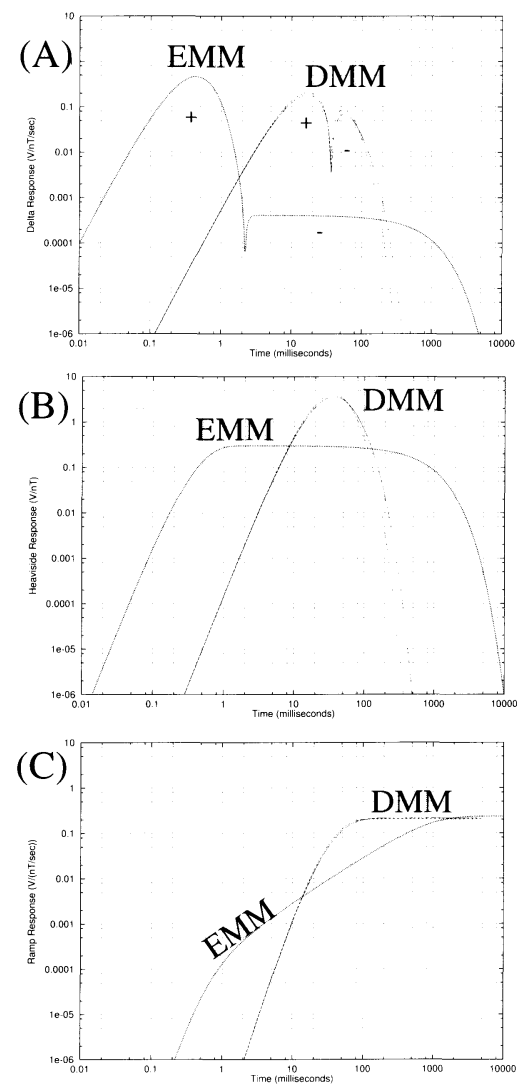

Fig. 3. The Impulse response $I(t)(A)$, the Heaviside response $\mathrm{H}(\mathrm{t})(\mathrm{B})$, the ramp response $\mathrm{P}(\mathrm{t})(\mathrm{C})$ for the DMM (NS: solid lines, EW: broken lines) and EMM (dotted lines).

$$
\begin{aligned}
& \Phi_{\tau_{\mathrm{r}}}[\mathrm{B}]= \int_{0}^{\infty} \mathrm{B}(\mathrm{t}-\xi)\left[\frac{\tau_{\mathrm{d}}^{3}}{\left(\tau_{\mathrm{d}}-\tau_{\mathrm{r}}\right)^{3}} \mathrm{e}^{-\xi / \tau_{\mathrm{r}}}\right. \\
&+\frac{\tau_{\mathrm{d}}^{2} \xi}{\left(\tau_{\mathrm{d}}-\tau_{\mathrm{r}}\right)^{2} \tau_{\mathrm{r}}} \mathrm{e}^{-\xi / \tau_{\mathrm{r}}} \\
&+\frac{1}{2} \frac{\tau_{\mathrm{d}}}{\left(\tau_{\mathrm{d}}-\tau_{\mathrm{r}}\right)}\left(\frac{\xi}{\tau_{\mathrm{r}}}\right)^{2} \mathrm{e}^{-\xi / \tau_{\mathrm{r}}} \\
&\left.+\frac{1}{6} \frac{\tau_{\mathrm{d}}}{\tau_{\mathrm{r}}}\left(\frac{\xi}{\tau_{\mathrm{r}}}\right)^{3} \mathrm{e}^{-\xi / \tau_{\mathrm{r}}}\right] \mathrm{d} \xi \\
& \Phi_{\tau_{\mathrm{d}}}[\mathrm{B}]=\int_{0}^{\infty} \mathrm{B}(\mathrm{t}-\xi) \frac{\tau_{\mathrm{d}}^{3}}{\left(\tau_{\mathrm{d}}-\tau_{\mathrm{r}}\right)^{3}} \mathrm{e}^{-\xi / \tau_{\mathrm{d}} \mathrm{d} \xi},
\end{aligned}
$$

and

$$
\mathrm{V}(\mathrm{t})=\mathrm{A} \frac{\left(\Phi_{\tau_{\mathrm{r}}}[\mathrm{B}]-\Phi_{\tau_{\mathrm{d}}}[\mathrm{B}]\right)}{\left(\tau_{\mathrm{d}}-\tau_{\mathrm{r}}\right)}
$$

Eqs. [9], [10] and [11], together with Fig. 3, imply that what DMM actually records is the difference between the B-value weighted for approximately 30 [ms] before the measurement (i.e., the duration of the positive lobe depicted in Fig. 3 (A) and the B-value weighted for approximately $100[\mathrm{~ms}]$ before the measurement (i.e., the duration of the negative lobe in Fig. 3 (A)) divided by an appropriate time difference. Thus, the DMM output approaches $200[\mathrm{mV}]$ per [nT/s] after 100 [ms] (see Fig. 3 (C)) from the initiation of a constantly increasing magnetic field variation. On the other hand, what an EMM records is the difference between the B-value weighted for approximately $1[\mathrm{~ms}]$ before the measurement and the B-value weighted for approximately $1[s]$ before the measurement (these values refer to the durations of the positive and negative lobe respectively, depicted in Fig. 3 (A) for EMM) divided by an appropriate time difference. Thus an EMM gives $\sim 220 \mathrm{mV}$ per nT/s after 1 [s] (see Fig. 3 (C)) from the initiation of a constantly increasing magnetic field variation.

Finally, Fig. 3 (B) shows that the output of DMM is "neutralized" (by falling almost three orders of magnitude) after $\approx 200[\mathrm{~ms}]$ from the arrival of a fast step magnetic variation, whereas the corresponding time for $\mathrm{EMM}$ is $\approx 5$ [s]; in other words, a signal recorded by DMM should correspond to a magnetic variation that has "arrived" at the sensor less than 200 [ms] before the recording.

Furthermore, note that for periods longer than around half a second, DMM operates essentially as $\mathrm{dB} / \mathrm{dt}$ detector; the same holds for EMM for periods longer than around 3 [s] (while for periods shorter than 3 [s], the latter dominantly acts as B-field detector, see $\mathrm{EMI}^{10)}$ ).

Examples of artificial noises and MT disturbances. Nearby artificial noises. Fig. 4 (A) depicts an example of a nearby artificial noise recorded by the MT-1 system of EMI with a sampling rate $\mathrm{F}_{\mathrm{s}}=8$ samples/sec, using the so-called remote reference technique. The latter involves simultaneous measurements of five components $\mathrm{E}_{\mathrm{NS}}, \mathrm{E}_{\mathrm{EW}}, \mathrm{B}_{\mathrm{NS}}, \mathrm{B}_{\mathrm{EW}}, \mathrm{B}_{\mathrm{z}}$ at two sites differing by a distance of the order of $\sim 10[\mathrm{~km}]$. The noise is recorded solely by the instrumentation installed at one of the two sites. The relevant recordings from such a site can be visualized in Fig. 4 (A). We note that the initiation of this disturbance is simultaneous (within experimental accuracy) at the electric and magnetic channels. This can be assured by calculating the cross-correlation coefficient (labeled "cr" hereafter) between the two-time series versus their time-lag: the first time-series corresponds to one of the two horizontal components of the electric field and the second to one of the three magnet- 


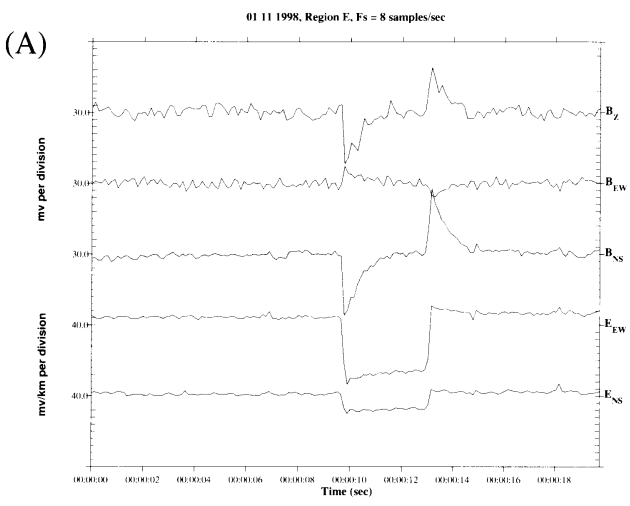

(B)

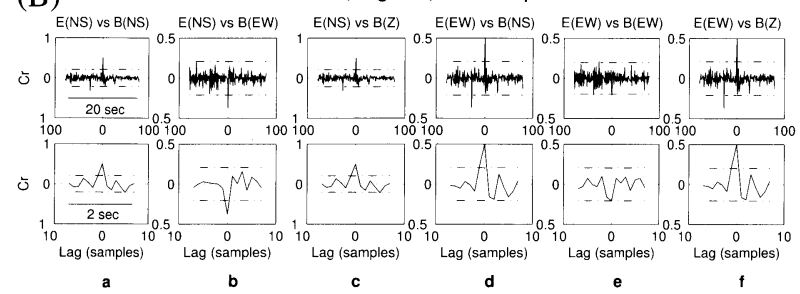

Fig. 4. Example of a nearby noise recorded at a site lying almost half a km south of region "C" of $\mathrm{IOA}^{2)}$ on Nov. 1, 1998, by the remote reference technique described in the text, using the MT1 system of EMI: (A) Recordings of the electric field (lower two channels) and the magnetic field (upper three channels) in an expanded time scale. The recordings correspond to 512 samples (per channel) collected with a rate $\mathrm{F}_{\mathrm{s}}=8$ samples/sec. (B) The corresponding values of "cr" (calculated after pre-whitening) between $\mathrm{E}$ and $\mathrm{B}$ versus the time lag. Dotted line indicates the $99 \%$ confidence interval.

ic components. The above behavior has also been assured when using DMM.

MT variations. Fig. 5 (A) shows an example of an MT disturbance simultaneously recorded on both types of coil magnetometers, i.e., DMM and EMM and collected by the permanently operating Campbel Datalogger $21 \mathrm{X}$ acquisition system $\left(\mathrm{F}_{\mathrm{s}}=1 \mathrm{sample} / \mathrm{sec}\right)$. The recordings of 11 (out of 16) channels are depicted as follows: the lowest three channels correspond to the three $\mathrm{DMM}$, labeled $\mathrm{B}(\mathrm{D})_{\mathrm{NS}}, \mathrm{B}(\mathrm{D})_{\mathrm{EW}}$ and $\mathrm{B}(\mathrm{D})_{\mathrm{Z}}$ respectively, and the next three channels to EMM (labeled with $\mathrm{E}$ in parentheses); the 7th and 8th channel correspond to two short electric dipoles (the subscript "c" stands for the site "C" of IOA depicted in Fig. 2(b) of Varotsos et al. ${ }^{2)}$ ), while the upper three channels correspond to the three long dipoles (see Fig. 2 (a) of Varotsos et al. ${ }^{2)}$ ). Fig. 5 (B) shows the calculated "cr" values between the electric field variations and the DMM recordings versus their time lag. An inspection of this figure reveals that the magnetic field recordings "precede" to a marked degree those of
(A)

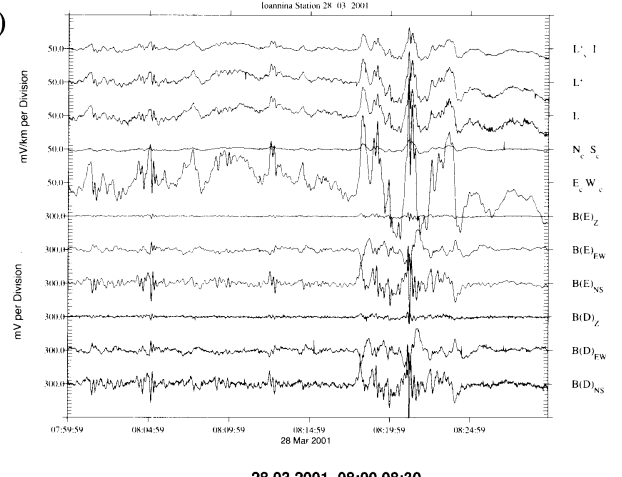

(B)
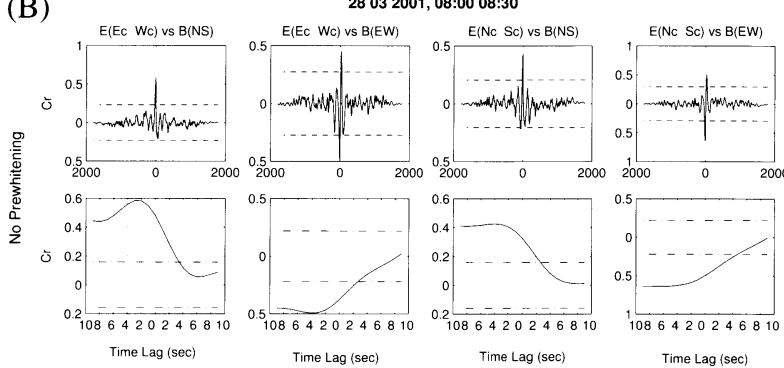

Fig. 5. (A) An example of MT disturbance recorded at IOA on March 28, 2001 by using both types of coil magnetometers (i.e., the lower three channels correspond to DMM, while the next three to EMM) and the permanently operating Datalogger $21 \mathrm{X}$ acquisition system $\left(\mathrm{F}_{\mathrm{s}}=1 \mathrm{sample} / \mathrm{sec}\right)$. (B) Calculated values of "cr" between one horizontal E-field component and one B-field component versus their time lag $\Delta \mathrm{t}$ (upper: $\Delta \mathrm{t}$ from-1000 to 1000 [s]; below: $\Delta \mathrm{t}$ from -10 to 10 [s]). Only the DMM recordings have been used in the calculation.

the electric field. This can be understood by recalling that, for the periods of the order of several seconds visualized in Fig. 5 (A), the DMM sensors act as dB/dt detectors as mentioned above in the calibration section. It might be noted that, in the case of plane waves in a uniformly conductive half-space, $\mathrm{E}_{\mathrm{x}}$ must lead $\mathrm{B}_{\mathrm{y}}$ in phase by $45^{\circ}$.

Conclusions. For the usual nearby artificial noise, the measured electric variations "arrive" simultaneously (i.e., with a time lag appreciably smaller than 1 [s]) with the magnetic ones detected either by DMM or EMM. On the other hand, for the usual MT disturbances (i.e., for periods larger than 1 [s]), the DMM recordings lead the electric ones by a measurable time lag.

Acknowledgements. We would like to express our gratitude to Prof. S. Uyeda, M. J. A., for very useful suggestions and critical reading of the ms. The collaboration of Prof. I. I. Rokityansky in designing the configuration of the field calibration measurements is greatly 
appreciated. Dr. A. Abdularazak, Mr. G. Tsoumanis and Mr. A. Varotsos participated in the latter measurements.

\section{References}

1) Varotsos, P., Sarlis, N., and Skordas, E. (2001) Proc. Japan Acad. 77B, 93-97.

2) Varotsos P., Lazaridou, M., Eftaxias, K., Antonopoulos, G., Makris, J., and Kopanas, J. (1996) In the Critical Review of VAN: Earthquake Prediction from Seismic Electric Signals (ed. Sir Lighthill, J.). World Scientific Publishing Co., Singapore, pp. 29-76.

3) Sarlis, N., Lazaridou, M., Kapiris, P., and Varotsos, P. (1999) Geoph. Res. Lett. 26, 3245-3248,.

4) Sarlis, N., and Varotsos, P. (2001) Acta Geophys. Pol. XLIX, 277-285.

5) Sarlis, N., and Varotsos, P. (2001) Journal of Geodynamics (to appear).

6) Varotsos, P., and Sarlis, N. (2001) In Ionospheric Electromagnetic Variations Related to Earthquakes (eds. Hayakawa, M., and Molchanov, O.). Terra Scientific Pub., Tokyo (to be published).

7) Varotsos, P., Eftaxias, K., Lazaridou, M., Nomicos, K., Bogris, N., Makris, J., Antonopoulos, G., and Kopanas, J. (1996) Acta Geophys. Pol. XLIV, 301-327.

8) Varotsos, P., Sarlis, N., Lazaridou, M., and Kapiris, P. (1996) Practika Athens Academy 71, 283-354.

9) Makris, J. (1997) Magnetotelluric inspection of Ioannina area. Ph.D. Thesis, University of Athens.

10) EMI (1995) Manual of MT-1. A mobile System for MT MeaSurements, Electro Magnetic Instruments, Berkeley, CA., p. 100.

11) Press, W. H., Teukolsky, S., Vettrling, W., and Flannery, B. P. (1992) Numerical Recipes in FORTRAN, Cambridge University Press, New York, pp. 688-693. 